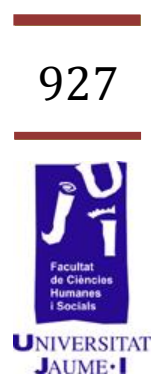

\title{
Arteterapia y educación emocional Mini Artistas: Viaje al centro del corazón
}

María Casabó Martín al239278@uji.es

Adriana García Soriano al087927@uji.es 
I. Resumen

Mangumen

En nuestro proyecto de innovación trabajamos el arte como terapia para fomentar la educación emocional puesto que creemos que esta técnica es un buen modo para trabajar las emociones en el aula.

El proyecto está dirigido al alumnado de Educación Infantil y a los profesionales que ejercen docencia en esta etapa educativa, de un centro educativo de la localidad de Burriana (Castellón)

Mediante ella, podemos conseguir el dominio de las habilidades emocionales y de esa manera, potenciar un desarrollo evolutivo y socioemocional adecuado del alumnado.

Lo que queremos conseguir mediante la arteterapia es el trabajo y la expresión de las emociones, sentimientos, pensamientos, frustraciones, miedos... para que el alumnado de Educación Infantil, a quién va dirigido este proyecto, sea capaz de reconocerlos y aprenda a gestionarse a sí mismo/a y a expresar las sensaciones que experimenta de forma verbal.

Todo ello se cuestiona para que el alumnado consiga ser más hábil socialmente y desarrollar su inteligencia emocional, así como el conjunto de habilidades sociales y necesarias para desenvolverse en la sociedad en la que vive.

Palabras clave: arteterapia, educación emocional, educación infantil

\section{Introducción}

El arte como terapia busca indagar en el conocimiento interno del ser a través de las emociones estéticas y su expresión, especialmente cuando el ejercicio de la palabra no basta o no es suficiente.

De acuerdo con la Asociación Americana de Arteterapia $^{1}:<<$ La arteterapia se basa en la creencia de que el proceso creativo desarrollado en la actividad artística es terapéutico y enriquecedor de la calidad de la vida de la persona>>.

Arteterapia es el uso terapéutico de la actividad artística en el contexto de una relación profesional por personas que experimentan enfermedades, dificultades en la vida, así como las personas que buscan un desarrollo personal ${ }^{2}$.

Según los autores Salovey i Mayer (1990) la Inteligencia Emocional es "la habilidad para gestionar los sentimientos y emociones propias y de los demás, de discriminar entre ellas y utilizar esta información para guiar el pensamiento y la acción".

La arteterapia en la educación con niños y niñas es de utilidad por tanto, para desarrollar su inteligencia emocional porque la creación artística les permite definirse y hacer frente a situaciones de angustia, en momentos críticos de su formación, de rechazo, y así poder hacer frente a

\footnotetext{
${ }^{1}$ American Association of Art Therapy

${ }^{2}$ Tema central del presente trabajo, la relación de la arteterapia con las emociones
} 
su dolor por medio del juego artístico. Esta técnica es un medio que se pone a disposición de los alumnos, como un juego nuevo, por medio de él podrán aprender a revivir situaciones pasadas, practicar y sentir la representación para poder dominarlas por medio de la simbolización.

La arteterapia propicia el encuentro con uno mismo/a y con las propias emociones, pudiendo así acceder a conflictos profundos que serían más difíciles de llegar a través de la palabra. También es un buen modo para favorecer la relación con los demás, desarrollando así la sociabilidad, permitiendo que nos expresemos de manera diferente $y$ enriquecedora.

En conclusión la arteterapia es una buena forma de conseguir fortalecer el desarrollo personal y social de las personas, por lo que en nuestra opinión es una manera idónea, para que los niños y niñas desarrollen la educación emocional adecuada.

\section{Objetivos}

La importancia de la arteterapia reside especialmente en el proceso de creación que se lleva a cabo, más que en el producto artístico que se obtiene. Esto es debido a que es durante dicho proceso cuando el niño/a aprende y se encuentra con situaciones frustrantes o de éxito que debe aprender a afrontar, pudiendo extrapolar éstas a otras áreas de su vida y ayudándole, por tanto, a buscar soluciones y aprendiendo a superar las dificultades y limitaciones que se pueda encontrar.

Además, en nuestro proyecto pretendemos desarrollar la competencia emocional, por lo que es interesante tener en cuanta que a través de la realización de las diferentes actividades artísticas, tanto individuales como grupales, el niño o niña experimenta gran variedad de emociones y sentimientos, por su cabeza pasan mil pensamientos... de modo que es una buena forma de educarse en el ámbito emocional y aprender a controlarlo y conducirlo del modo adecuado.

Por tanto nuestro objetivo general será conseguir mediante la arteterapia el trabajo y la expresión de las emociones, sentimientos, pensamientos, frustraciones, miedos... para que cada niño/a sea capaz de reconocerlas/os y aprenda a gestionarse a si mismo/a y a expresar las sensaciones que experimenta de forma verbal, de modo que consiga ser más hábil socialmente y desarrollar su inteligencia emocional, así como el conjunto de habilidades sociales necesarias para desenvolverse en la sociedad en la que vive.

Para acotar mejor nuestro trabajo se concretan, a continuación, algunos objetivos específicos que queremos llevar a cabo:

- Aprovechar las sensaciones que se generen a través del trabajo plástico para realizar trabajos creativos que surjan de las propias emociones y sentimientos de los niños/as. 
- Potenciar la capacidad creativa de cada uno de los alumnos/as.

- Generar el espacio adecuado para la socialización de la experiencia individual, tanto en lo creativo como en lo personal.

- Posibilitar que los niños/as trabajen y experimenten con diferentes materiales y al mismo tiempo, sean capaces de transmitir sus emociones, sentimientos, preocupaciones...

\section{Material y método}

El colegio que se seleccionó para llevar a cabo la presente investigación, fue un colegio concertado de Burriana y los criterios de selección que seguimos fueron varios, entre ellos el hecho de que una de las investigadoras trabaja en él, por lo que nos resultaría más fácil la comunicación y acceso a éste.

Por otro lado, dada la necesidad existente por parte de los niños y niñas de estas edades de aprender a conocerse a sí mismos, a comprender sus reacciones ante las situaciones que viven día a día, a reconocer e identificar cada una de sus emociones y sentimientos, también a comprender a los demás, a ser mejores compañeros/as, a resolver los conflictos mediante el respeto a los pensamientos y sentimientos de la otra persona...es importante trabajar todos estos factores desde pequeños/as ya que les ayudará a crecer y desarrollarse de forma sana y más feliz, reduciendo las frustraciones y ayudándoles a ir adquiriendo las habilidades sociales tan necesarias para desenvolverse en la sociedad actual.

Otros factores que influyeron en esta decisión es que el centro seleccionado, no lleva a cabo ningún Proyecto de Innovación Curricular, por lo que sería meramente interesante ofrecerles la posibilidad de vivenciar la experiencia. Si pudiéramos llevar a cabo nuestra propuesta de proyecto, conseguiríamos que perdieran el miedo a implantar cosas nuevas viendo los beneficios y mejoras que conseguirían mediante esta forma de trabajo.

A la hora de la elaboración del cuestionario, que se presenta en la Tabla 1, puesto que nuestro proyecto va dirigido a la etapa de educación infantil, decidimos que la muestra estaría compuesta por los maestros y maestras de dicha etapa. Al mismo tiempo, se creyó conveniente pasarlo al director del centro porque como tal debería estar al tanto del tipo de proyecto que se iba a realizar en su centro, así como a la psicopedagoga porque aunque no ejerza docencia directa con este alumnado también desempeña sus funciones en esta etapa educativa. Por otro lado se creyó interesante pasar el cuestionario a la maestra de audición y lenguaje y a la maestra de pedagogía terapéutica ya que son dos profesionales que trabajan en esta etapa y pretendemos que participen activamente. 
En definitiva la muestra estaba compuesta por 10 profesionales del centro: 6 maestros y maestras de Educación Infantil, el Director del Centro, la Psicopedagoga, la Maestra de Audición y Lenguaje y la Maestra de Pedagogía Terapéutica.

El cuestionario era totalmente anónimo y estaba compuesto por 13 ítems. En primer lugar elaboramos un gran número de ítems, que posteriormente resumimos en 13 ya que englobamos unos en otros $y$ eliminamos algunos que no nos ofrecían información relevante, con la finalidad de no hacer un cuestionario demasiado extenso. Con esta medida pretendíamos que no resultara pesado contestarlo y que, por tanto, consiguiéramos un porcentaje de fiabilidad mayor.

Los ítems estaban planteados mediante preguntas directas de respuesta "si" o "no" y otros ítems de respuesta abierta para que los encuestados pudiesen responder libremente.

Tras la elaboración del cuestionario, nos pudimos en contacto con el director del centro para exponer el objetivo de nuestro trabajo y le mostramos el cuestionario que pretendiamos pasar. Tras recibir la aprobación de dirección entregamos dichos cuestionarios, que fueron recogidos días después respondidos por la totalidad de la muestra

Tabla 1. Ítems del cuestionario

\begin{tabular}{|c|}
\hline EVALUACIÓN INICIAL \\
\hline Centro: ................................................ Población: ....................... \\
\hline Sexo \\
\hline 1. ¿Has participado y/o realizado un proyecto de innovación? \\
\hline 2. En caso afirmativo, ¿en qué consistía el proyecto? ¿De qué tema trataba? \\
\hline 3. Nivel educativo al que perteneces \\
\hline 4. ¿Qué necesidades presenta tu alumnado? \\
\hline 5. ¿Conoces el concepto arteterapia? \\
\hline $\begin{array}{l}\text { 6. ¿Cómo crees que se puede aplicar la arteterapia en tus alumnos, en relación al trabajo de las } \\
\text { emociones? }\end{array}$ \\
\hline 7. ¿Crees que es beneficioso trabajar mediante la arteterapia la educación emocional? \\
\hline $\begin{array}{l}\text { 8. En caso afirmativo, ¿qué beneficios crees que puede aportar el trabajo de la arteterapia en } \\
\text { tus alumnos? }\end{array}$ \\
\hline $\begin{array}{l}\text { 9. ¿Crees que dispones de los recursos necesarios para poder realizar el proyecto de } \\
\text { innovación? }\end{array}$ \\
\hline 10. En caso afirmativo, ¿cuáles son? \\
\hline $\begin{array}{l}\text { 11. En caso negativo, ¿qué recursos crees que son necesarios? Indica tanto los humanos, como } \\
\text { los materiales. }\end{array}$ \\
\hline $\begin{array}{l}\text { 12. ¿Te gustaría participar con tu alumnado en un proyecto sobre la arteterapia y la educación } \\
\text { emocional? }\end{array}$ \\
\hline OBSERVACIONES \\
\hline
\end{tabular}


V. Resultados

A continuación vamos a mostrar los resultados obtenidos a partir de nuestro cuestionario, destacando los más relevantes para nuestra investigación y presentando los resultados basándonos en la totalidad de las personas encuestadas, sin diferenciar entre unos $y$ otros profesionales.

De este modo comprobamos que nuestra muestra está compuesta por 10 personas, en concreto 2 hombres y 8 mujeres. De ellos/as, el 20\% afirman haber participado en algún proyecto de Innovación Curricular, mientras que el resto, es decir un $80 \%$ de ellos, nunca han participado. Como se muestra en la Figura 1, los proyectos de Innovación en los cuales ha participado el $20 \%$ de la muestra son los siguientes.

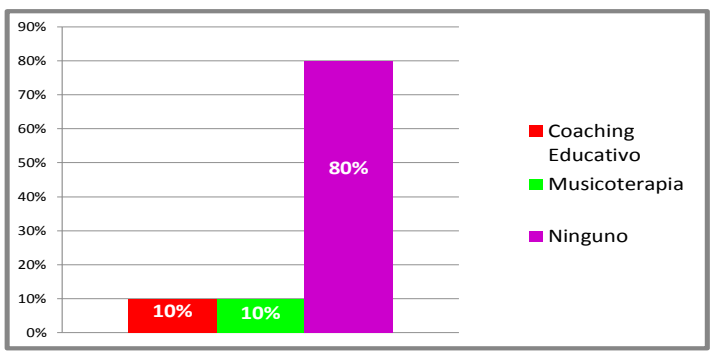

Figura 1. Proyectos en los que han participado

En la Figura 2 encontramos el ítem 5, tratándose de una pregunta abierta con la que se pretende averiguar cuáles son las necesidades que presenta el alumnado con el que trabajan diariamente la muestra de encuestados. En la siguiente figura se muesytran cuáles son los resultados obtenidos.

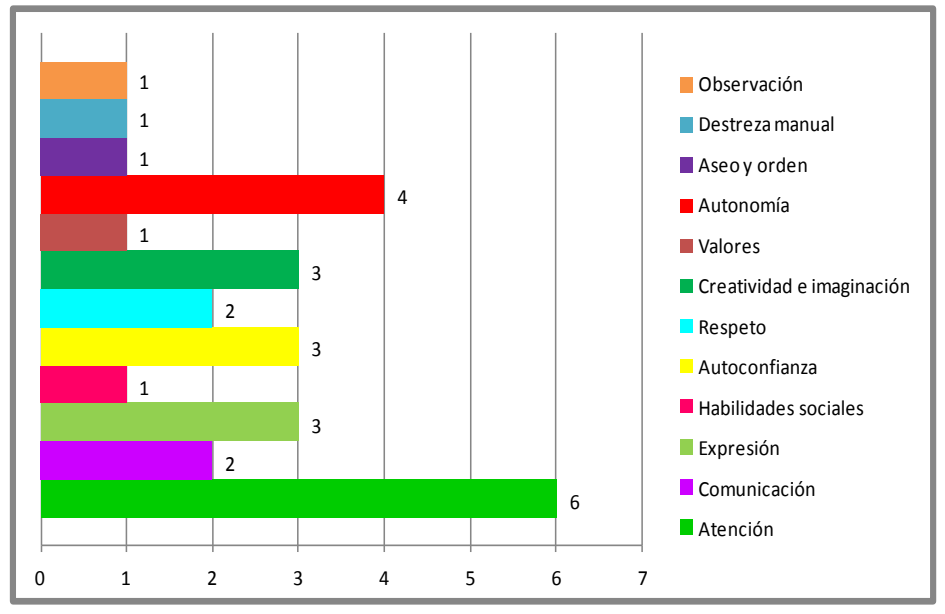

Figura 2. Necesidades que presenta el alumnado 
De este modo observamos claramente que existe una gran diversidad de necesidades, y que muchas de ellas, están estrechamente relacionadas con las destrezas que se pretenden desarrollar a través de nuestro proyecto, como las habilidades sociales, la autonomía y la autoconfianza, entre otros.

En el siguiente ítem obtenemos que el $80 \%$ de la muestra tiene conocimiento de qué es la arteterapia, hecho que nos resulta positivo puesto que así sus diferentes respuestas sobre el tema tienen mayor fiabilidad $y$, este aspecto facilitaría la posterior puesta en práctica de dicho proyecto de innovación educativa.

El ítem 7 pretende conocer si la muestra conocía las posibles aplicaciones que se le pueden dar a la arteterapia con respecto a los alumnos de infantil, en relación al trabajo de las emociones.

En la Figura 3, que presentamos a continuación, recopila los diferentes aspectos que la muestra cree que se podrían trabajar a partir de la aplicación de un proyecto de estas características.

Como se puede observar, los resultados nos muestran una gran variedad de posibilidades de aplicación de la arteterapia en el aula y cabe señalar que sus aportaciones han sido nombrosas y muestran una gran diversidad. Las aplicaciones que consideramos más relevantes son: seguridad afectiva, la reflexión, la autoestima, salud y caldiad de vida, valoración de las emociones, autoconocimiento y expresión emocional. De ahí, la importancia de este ítem para reforzar y dar más hincapié a estos factores durante la realización del proyecto.

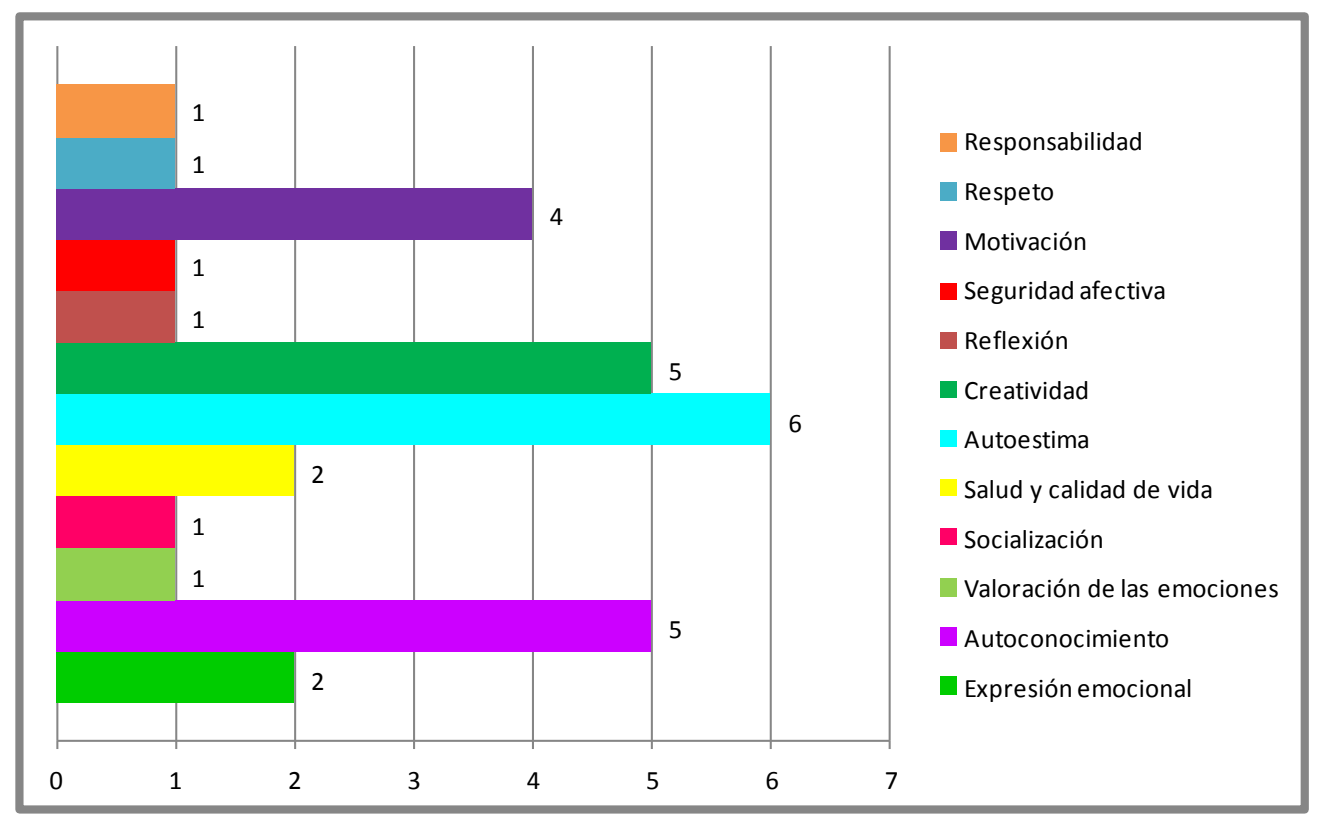

Figura 3. Aplicación de la arteterapia

En el ítem 8, obtenemos que el $100 \%$ de la muestra opina que el trabajo de la educación emocional a través de la arteterapia es un proyecto que resultaría beneficioso para el desarrollo y crecimiento del alumnado, no sólo para el autoconocimiento, sino también para conocer a los compañeros/as. 
Partir de la totalidad del acuerdo en este ítem es muy positivo, puesto que es primordial contar con el consentimiento y aprobación de todas las personas que van a estar en contacto con los niños y niñas de esta etapa y que van a participar activamente en él, ya que así, podemos conseguir realizarlo con la mayor motivación y participación posible.

En la Figura 4 se detallan los beneficios específicos que la muestra piensa que obtendría el alumnado mediante el trabajo de la emoción a través de la arteterapia. Al tratarse de una pregunta abierta, como se observa en la figura, existe una gran variedad de beneficios que puede aportarnos la arteterapia centrándola en la educación emocional.

Por tanto, teniendo en cuenta la gran variedad de beneficios que se pueden obtener trabajando las emociones mediante esta técnica, se cree conveniente e interesante poder llevar a cabo dicho proyecto para mejorar así la educación emocional de los destinatarios del mismo.

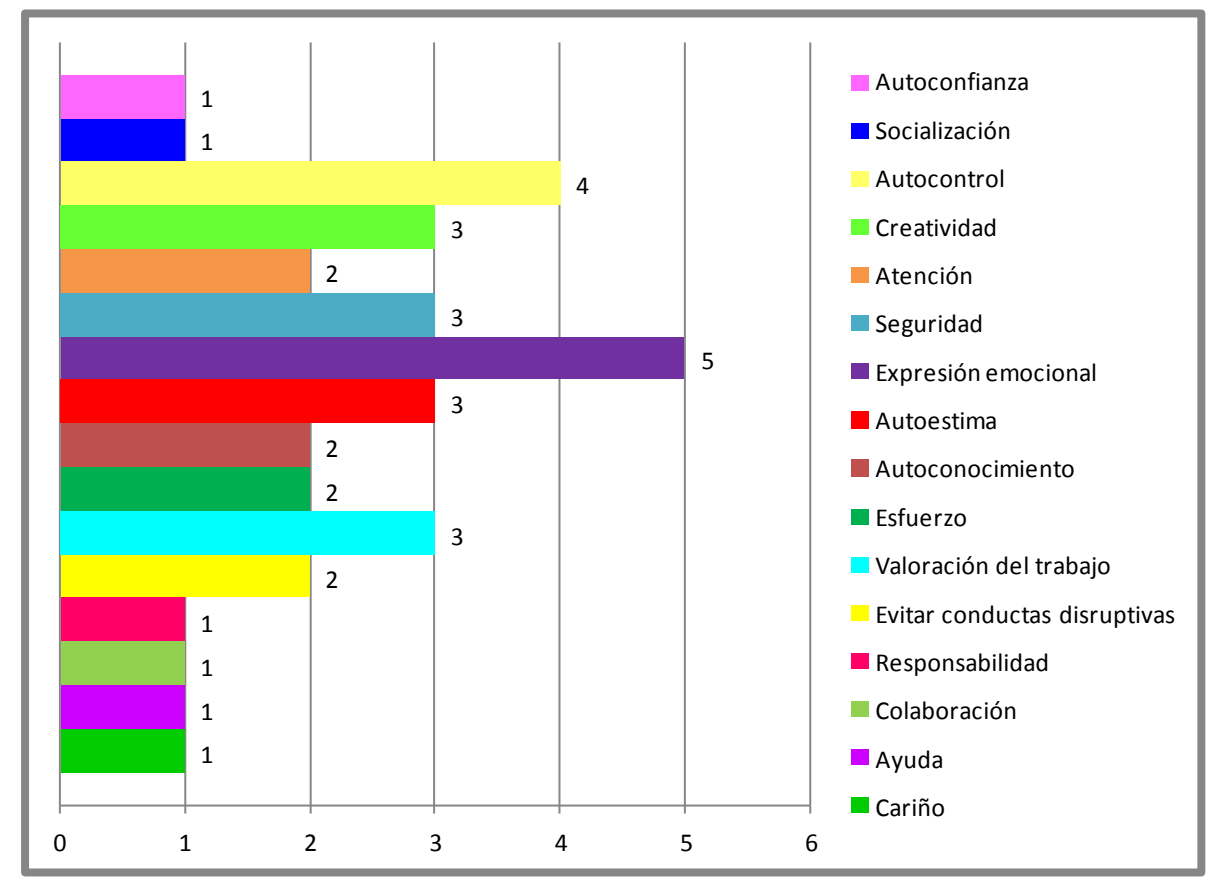

Figura 4. Beneficios de la arteterapia

Analizando los resultados del cuestionario, se observa que el $70 \%$ de los encuestados opinan que el centro en el que trabajan disponen de los recursos necesarios para llevar a cabo un proyecto de las características que les presentamos, por ello se propone otro ítem que han de responder especificando el material del que disponen.

A partir de éste obtenemos los resultados siguientes, que se observan en la Figura 5. 


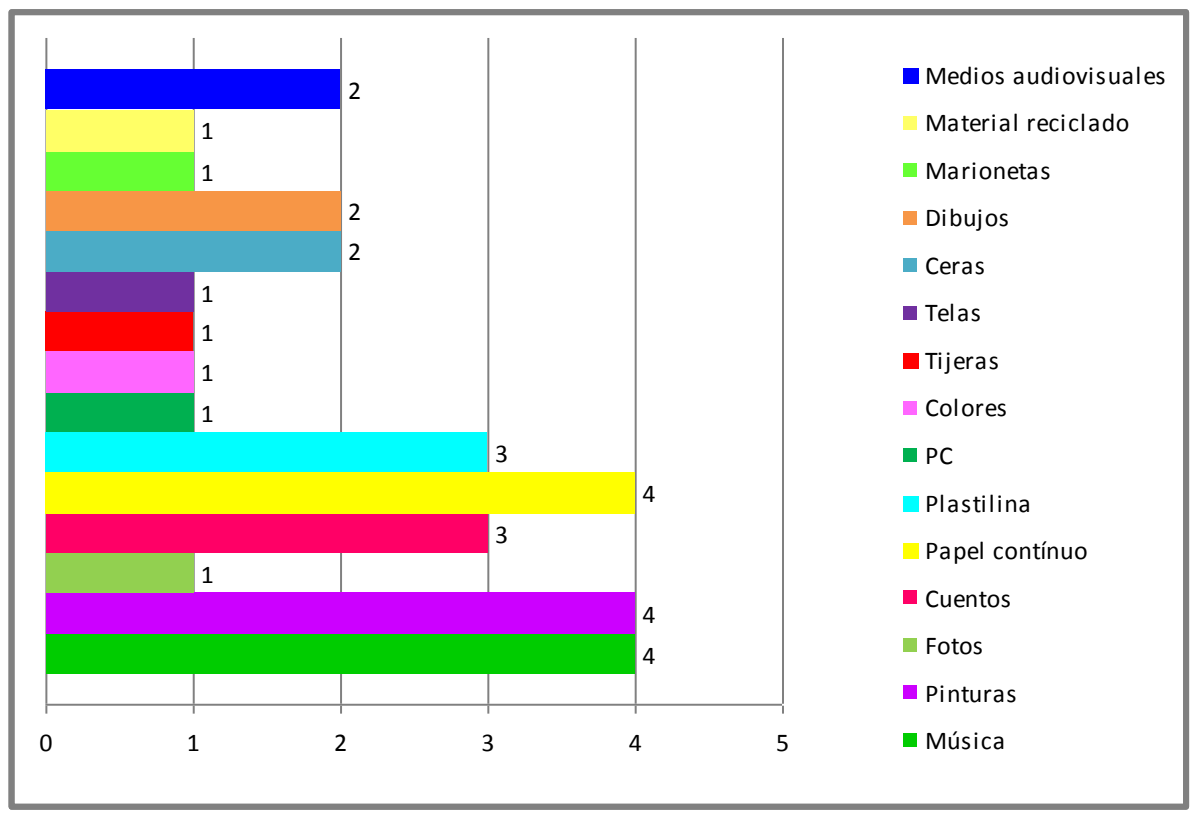

935

Material reciclado

Marionetas

Dibujos

Ceras

- Telas

Tijeras

IVERSITAT

JAUME-I

Figura 5. Recursos disponibles

A pesar de la discrepancia entre unas opiniones y otras en el ítem anterior, podemos observar que el centro dispone de varios recursos y de gran variedad de materiales, que pueden ser interesantes y útiles. Entre ellos encontramos las pinturas, las fotos, el papel continuo, el material reciclado... Aunque algunos no sean específicamente materiales para trabajar el arte creemos que se puede sacar partido de ellos y realizar actividades muy interesantes a partir de estos recursos.

En el ítem 12 reflejado en la Figura 6, pretendíamos averiguar qué recursos son aquellos con los que debería contar el centro para poder realizar, de la mejor manera posible, las sesiones de arteterapia con los alumnos y alumnas de educación infantil.

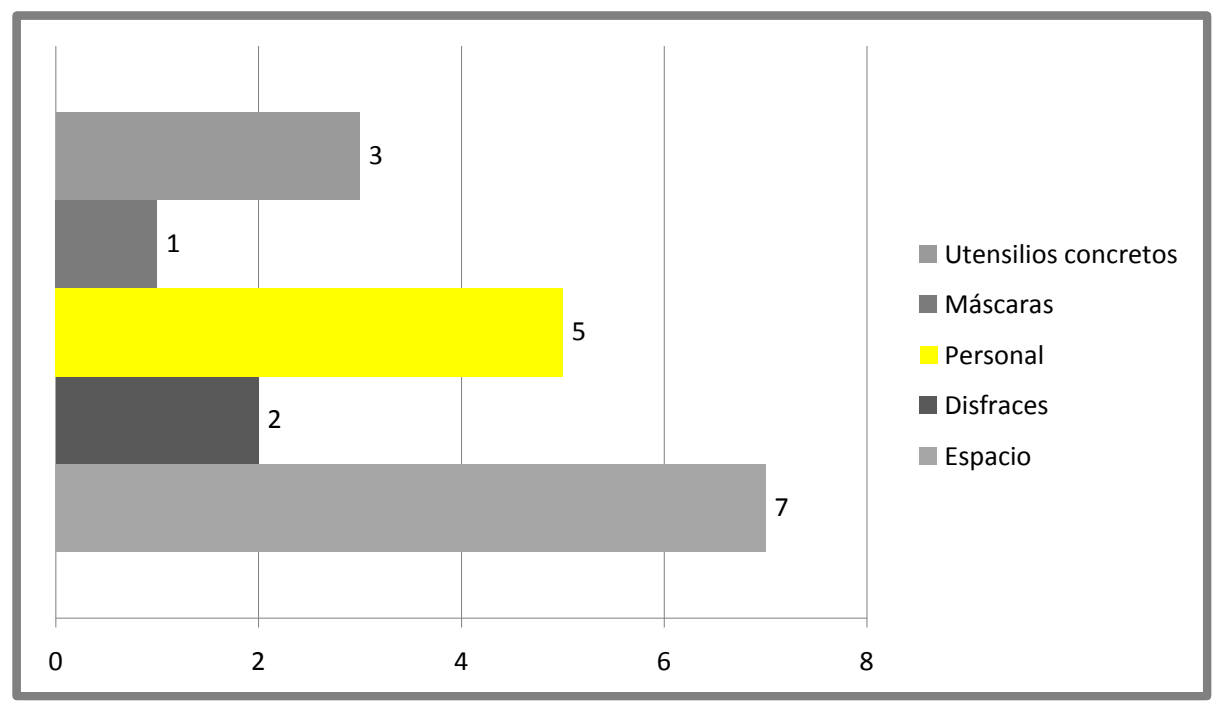

Figura 6. Recursos necesarios 
Como podemos observar, pese que un $70 \%$ de maestros y maestras y otros profesionales del centro opinan que cuentan con adecuados $y$ suficientes materiales para poder realizar las actividades que serían necesarios, en este ítem la mayoría coincide en la falta de espacio necesario para poder realizar de la mejor manera posible dichas actividades así como que los profesionales con los que cuentan son también insuficientes para realizarlo, y carecen de la formación necesaria.

Finalmente, presentamos un último ítem a través del cual queríamos saber si a la muestra encuestada participaría en el proyecto de innovación educativa que proponemos, con el fin de mejorar el desarrollo de la educación emocional de todos los implicados en éste, tanto alumnos como profesionales.

Obtenemos que el $100 \%$ participarían en el proyecto hecho que nos resulta muy positivo.

Tras el análisis de los resultados de nuestro cuestionario cabe destacar que nuestro proyecto no pudo llevarse a cabo en dicho centro, puesto que en el momento de ponerlo en marcha se presentaron diversos problemas que impidieron que esto sucediera.

\section{Discusión y conclusiones}

Una vez realizado el proyecto de innovación educativa cabe hacer hincapié en la importancia de la arteterapia, puesto que a partir de esta técnica se pueden trabajar las emociones.

Como bien hemos podido comprovar la arteterapia puede verse como un tipo especial de aprendizaje que trata con el mundo interno de las personas, el yo mismo, como con su mundo social inmediato, los otros y yo.

Es por tanto, una técnica que puede tener una gran relevancia en los centros educativos para el trabajo de las emociones ya que ayuda a desarrolar y valorar todos aquellso aspectos que se consideran de gran importancia en la educación integral de los niños y de las niñas: las relaciones interpersonales, el bienestar personal, la autoestima, la comunicación, el auto-concocimiento...

Centrándonos en los resultados obtenidos a partir de los cuestionarios se puede decir, por tanto, que en este centro educativo creen que puede resultar productivo a la par que interesante y motivante, no sólo para el alumnado sino para todos los profesionales, el hecho de llevar a cabo un proyecto de innovación educativa de estas características, puesto que de una manera creativa y alegre los más pequeños pueden ir creciendo emocionalmente a la vez que se enriquecen con los contenidos que aprenden en el proceso de enseñanzaaprendizaje.

Como ya se ha comentado en el apartado anterior, el proyecto de innovación educativa objeto de nuestra investigación no pudo llevarse a cabo en el centro al cuál estaba dirigido, por ello, no podemos obtener 
unas conclusiones exactas y concisas de lo que sería el resultado final de nuestro proyecto.

A pesar de no poder llevar a cabo el proyecto anterior, el hecho de haberlo diseñado ha ayudado a conocer realmente como funciona un Proyecto de Innovación Educativa.

El elegir como tema para el proyecto la Arteterapia ha permitido profundizar más en el tema y sobre todo, saber cómo se pueden, a partir de la creatividad de los alumnos/as y de la expresión artística, trabajar muchos y diversos aspectos que abarcan las diferentes áreas y que se deben tener presentes en el proceso de enseñanza-aprendizaje.

Al mismo tiempo, se ha tomado mayor conciencia de los beneficios que conlleva recibir una educación emocional, basada en la expresión de los sentimientos, en la comprensión, en el respeto... ya que no se debe olvidar que todos somos personas que necesitamso desarrollarnos emocionalmente, a la par que formarnos en conocimientos.

\section{Bibliografía}

BATLLE, S.: Arte-Terapia. Cómo poner magia, colores y creatividad a cada día. Ediciones Obelisco.

SÁNCHEZ, J.: Enseñar arte es hacer feliz. Ediciones Mágina. Octaedro. Andalucía.

KLEIN, J.: Arterapia. Una introducción. Octaedro. 
